\title{
Unique Fixed-Point Results in Fuzzy Metric Spaces with an Application to Fredholm Integral Equations
}

\author{
Iqra Shamas, ${ }^{1}$ Saif Ur Rehman $\mathbb{D}^{1}{ }^{1}$ Hassen Aydi $\mathbb{D},{ }^{2,3,4}$ Tayyab Mahmood, ${ }^{5}$ \\ and Eskandar Ameer $\mathbb{D i D}^{6}$ \\ ${ }^{1}$ Department of Mathematics, Gomal University, Dera Ismail Khan 29050, Pakistan \\ ${ }^{2}$ Université de Sousse, Institut Supérieur d'Informatique et Des Techniques de Communication, H. Sousse 4000, Tunisia \\ ${ }^{3}$ Department of Mathematics and Applied Mathematics, Sefako Makgatho Health Sciences University, Ga-Rankuwa, South Africa \\ ${ }^{4}$ China Medical University Hospital, China Medical University, Taichung 40402, Taiwan \\ ${ }^{5}$ Department of Mathematics, COMSATS University Islamabad, Wah Cantt. 47040, Pakistan \\ ${ }^{6}$ Department of Mathematics, Taiz University, Taiz 6803, Yemen
}

Correspondence should be addressed to Hassen Aydi; hassen.aydi@isima.rnu.tn and Eskandar Ameer; eskandarameer@gmail.com

Received 21 April 2021; Accepted 1 September 2021; Published 23 September 2021

Academic Editor: Anita Tomar

Copyright (C) 2021 Iqra Shamas et al. This is an open access article distributed under the Creative Commons Attribution License, which permits unrestricted use, distribution, and reproduction in any medium, provided the original work is properly cited.

This paper aims at proving some unique fixed-point results for different contractive-type self-mappings in fuzzy metric spaces by using the "triangular property of the fuzzy metric". Some illustrative examples are presented to support our results. Moreover, we present an application by resolving a particular case of a Fredholm integral equation of the second kind.

\section{Introduction}

In 1922, Banach [1] proved a "Banach contraction principle (BCP)," which is stated as "a self-mapping in a complete metric space satisfying a contraction condition has a unique fixed point". This theorem plays a very important role in the theory of fixed points. Many researchers gave generalization and improved the BCP in many directions for single-valued and multivalued mappings in the context of metric spaces by ensuring the existence of fixed point, common fixed point, and coincidence point results with different types of applications, such as differential-type applications, integral-type applications, functional-type applications. In 2004, Ran and Reurings [2] proved a fixed-point theorem in a metric space by using partially ordered sets and they present some applications to matrix equations. While in [3], Nieto and Rodrguez-López extended and improved the result of Ran and Reurings [2] by using increasing mappings and applied the result to get a unique solution for the first-order ordinary differential equation with periodic boundary equations. In 2017, Priskillal and Thangavelu [4] established some fixed- point theorems in complete metric spaces by using $\psi$-contractive fuzzy mappings with an application to fuzzy differential equations. Some more fixed-point results in the context of metric spaces can be found in [5-16].

In 1965, the theory of fuzzy sets was introduced by Zadeh [17]. Lately, this theory is improved, investigated, and applied in many directions. Among them, we state the theory of fuzzy logic, which is based on the notion of relative graded memberships, as inspired by the processing of human perceptions and cognitions. Fuzzy logic can deal with information arising from computational perceptions and cognitions, that is, uncertain, obscure, imprecise, partly true, or without sharp limits. A fuzzy logic permits the inclusion of vague human assessments in computing problems. The fuzzy logic is extremely useful for many people associated with innovative work including engineering (electrical, chemical, civil, environmental, mechanical, industrial, geological, etc.), mathematics, computer software, earth science, and physics. Some of their findings can be found in [18-25].

The other direction of fuzzy sets is used in topology and analysis by many mathematicians. Subsequently, several 
authors have applied various forms of general topologies and developed the concept of fuzzy spaces. Kramosil and Michalek [26] developed the concept of a fuzzy metric space (FMspace). Later on, Grabeic [27] extended the BCP and proved a fixed-point result in FM-spaces in the sense of Kramosil and Michalek. George and Veeramani [28] modified the concept of FM-spaces with the help of continuous $t$-norms and proved some basic properties in this direction. In 2002, Gregori and Sapena [29] proved some contractive-type fixed-point theorems in complete FM-spaces in the sense of Kramosil and Michalek [26] and in the sense of George and Veeramani [28]. Rana et al. [30] established some fixed-point theorems in FM-spaces by using implicit relations. Many authors have introduced the number of fixed-point theorems in FMspaces by using the concept of compatible maps, implicit relations, weakly compatible maps, and R-weakly compatible maps (see [31-38] and the references therein). Furthermore, Beg and Abbas [39], Popa [40], and Imad et al. [41, 42] obtained some fixed-point and invariant approximation results in FM-spaces. Recently, Li et al. [43] proved some strong coupled fixed-point theorems in FM-spaces with an integral-type application. Later on, Rehman et al. [44] proved some rational fuzzy-contraction theorems in FM-spaces with nonlinear integral-type application.

The purpose of this paper is at obtaining some extended unique fixed-point theorems in FM-spaces without the "assumption that all the sequences are Cauchy" by using the concept of Li et al. [43] and Rehman et al. [44]. We present some illustrative examples and an integral-type application to support our work. By using this concept, one can prove more generalized contractive-type fixed-point and common fixed-point results in FM-spaces with different types of integral equations. Our paper is organized as follows: Section 2 consists of preliminary concepts. In Section 3, we prove some generalized fixed-point results without continuity in FMspaces and we presented some examples in the support of our obtained results. In Section 4, we consider some generalized Ćirić fuzzy contraction results in complete FM-spaces. In Section 5, we present an application of a particular case of the Fredholm integral equation of the second kind by ensuring the existence of a solution.

\section{Preliminaries}

The concept of a continuous $t$-norm is given by Schweizer and Sklar [45].

Definition 1 (see [45]). An operation * : $[0,1] \times[0,1] \longrightarrow$ $[0,1]$ is known as a continuous $t$-norm if it satisfies the following:

(1) * is commutative, associative, and continuous

(2) $1 * \rho_{1}=\rho_{1}$ and $\rho_{1} * \rho_{2} \leq \rho_{3} * \rho_{4}$, whenever $\rho_{1} \leq \rho_{3}$ and $\rho_{2} \leq \rho_{4}$, for all $\rho_{1}, \rho_{2}, \rho_{3}, \rho_{4} \in[0,1]$

The basic continuous $t$-norms: the minimum, the Lukasiewicz, and the product $t$-norms are defined, respectively, as follows:

$$
\begin{gathered}
\rho_{1} * \rho_{2}=\min \left\{\rho_{1}, \rho_{2}\right\}, \\
\rho_{1} * \rho_{2}=\max \left\{\rho_{1}+\rho_{2}-1,0\right\}, \\
\rho_{1} * \rho_{2}=\rho_{1} \rho_{2} .
\end{gathered}
$$

Definition 2 (see [28]). A 3-tuple $\left(W, M_{F}, *\right)$ is said to be a FM-space if $W$ is an arbitrary set, $*$ is a continuous $t$ -norm, and $M_{F}$ is a fuzzy set on $W \times W \times(0, \infty)$ satisfying the following:

(i) $M_{F}(w, x, t)>0$ and $M_{F}(w, x, t)=1 \Leftrightarrow w=x$

(ii) $M_{F}(w, x, t)=M_{F}(w, x, t)$

(iii) $M_{F}(w, z, t) * M_{F}(z, x, s) \leq M_{F}(w, x, t+s)$

(iv) $M_{F}(w, x, t):(0, \infty) \longrightarrow[0,1]$ is continuous for all $w, x, z \in W$ and $t, s>0$

Definition 3 (see $[28,29])$. Let $\left(W, M_{F}, *\right)$ be a FM-space, $w \in W$, and $\left\{w_{i}\right\}$ be a sequence in $W$. Then,

(i) A sequence $\left\{w_{i}\right\}$ in $W$ is said to be convergent to a point $w \in W$ if $\lim _{i \rightarrow \infty} M_{F}\left(w_{i}, w, t\right)=1$ for $t>0$

(ii) $\left\{w_{i}\right\}$ is said to be a Cauchy sequence, if for each 0 $<\varepsilon<1$ and $t>0$, there is $i_{0} \in \mathbb{N}$ such that $M_{F}\left(w_{k}\right.$, $\left.w_{i}, t\right)>1-\varepsilon, \forall k, i \geq i_{0}$

(iii) $\left(W, M_{F}, *\right)$ is complete, if every Cauchy sequence is convergent in $W$

(iv) $\left\{w_{i}\right\}$ is known as a fuzzy contractive, if there is 0 $<\beta<1$ so that

$$
\begin{aligned}
& \frac{1}{M_{F}\left(w_{i}, w_{i+1}, t\right)}-1 \\
& \quad \leq \beta\left(\frac{1}{M_{F}\left(w_{i-1}, w_{i}, t\right)}-1\right), \quad \text { for } t>0 \text { and } i \geq 1
\end{aligned}
$$

Definition 4 (see [46]). Let $\left(W, M_{F}, *\right)$ be a FM-space. The fuzzy metric $M_{F}$ is triangular if

$$
\frac{1}{M_{F}(w, x, t)}-1 \leq\left(\frac{1}{M_{F}(w, y, t)}-1\right)+\left(\frac{1}{M_{F}(y, x, t)}-1\right)
$$

for all $w, x, y \in W$ and $t>0$.

Lemma 5. A fuzzy metric $M_{F}$ is triangular. 
Proof. Let $M_{F}: W \times W \times(0, \infty) \longrightarrow[0,1]$ be a fuzzy metric defined by

$$
M_{F}(w, x, t)=\frac{t}{t+|w-x|}, \quad \text { for } w, x \in W \text { and } t>0 \text {. }
$$

Now, we have

$$
\begin{aligned}
\frac{1}{M_{F}(w, x, t)}-1 & =\frac{|w-x|}{t}=\frac{|w-z+z-x|}{t} \\
& \leq \frac{|w-z|}{t}+\frac{|z-x|}{t} \\
& =\left(\frac{1}{M_{F}(w, z, t)}-1\right)+\left(\frac{1}{M_{F}(z, x, t)}-1\right) .
\end{aligned}
$$

This implies that

$$
\begin{aligned}
\frac{1}{M_{F}(w, x, t)}-1 \leq & \left(\frac{1}{M_{F}(w, z, t)}-1\right) \\
& +\left(\frac{1}{M_{F}(z, x, t)}-1\right), \quad \text { for } t>0 .
\end{aligned}
$$

Hence, it is proved that a fuzzy metric $M_{F}$ is triangular.

Lemma 6 (see [46]). Let $\left(W, M_{F}\right.$ *) be a FM-space. Let $w$ $\in W$ and $\left\{w_{i}\right\}$ be a sequence in $W$. Then, $w_{i} \longrightarrow w$ iff $\lim _{i \rightarrow \infty} M_{F}\left(w_{i}, w, t\right)=1$, for $t>0$.
Definition 7 (see [29]). Let $\left(W, M_{F}, *\right)$ be a FM-space and $G: W \longrightarrow W$. Then, $G$ is known as a fuzzy contraction, if there is $0<h<1$ so that

$$
\frac{1}{M_{F}(G w, G x, t)}-1 \leq h\left(\frac{1}{M_{F}(w, x, t)}-1\right),
$$

for all $w, x \in W$ and $t>0$.

\section{Generalized Fixed-Point Results in FM- Spaces}

In this section, we consider some generalized contraction theorems on FM-spaces for fixed points (by using the "triangular property of the fuzzy metric").

Theorem 8. Let $\left(W, M_{F}, *\right)$ be a complete FM-space so that the fuzzy metric $M_{F}$ is triangular. Let $G: W \longrightarrow W$ satisfy

$$
\begin{aligned}
& \frac{1}{M_{F}(G w, G x, t)}-1 \\
& \leq a\left(\frac{1}{M_{F}(w, x, t)}-1\right)+b\left(\frac{1}{M_{F}(w, G w, t)}-1\right. \\
& \left.\quad+\frac{1}{M_{F}(x, G x, t)}-1+\frac{1}{M_{F}(x, G w, t)}-1+\frac{1}{M_{F}(w, G x, t)}-1\right) \\
& \quad+c\left(\frac{1}{\min \left\{M_{F}(w, G w, t), M_{F}(x, G x, t), M_{F}(x, G w, t), M_{F}(w, G x, t)\right\}}-1\right),
\end{aligned}
$$

for all $w, x \in W, a \in(0,1), b \in[0,1 / 4)$, and $c \in[0,1)$ with $(a+4 b+2 c)<1$. Then, $G$ has a unique fixed point.

Proof. Fix $w_{0} \in W$. Take an iterative sequence $\left\{w_{i}\right\}$ such that $w_{i+1}=G w_{i}$ for all $i \geq 0$. Now, by view of (8), we have

Then, we have for $t>0$,

$$
\begin{aligned}
\frac{1}{M_{F}\left(w_{i}, w_{i+1}, t\right)}-1= & \frac{1}{M_{F}\left(G w_{i-1}, G w_{i}, t\right)}-1 \leq a\left(\frac{1}{M_{F}\left(w_{i-1}, w_{i}, t\right)}-1\right)+b\left(\frac{1}{M_{F}\left(w_{i-1}, G w_{i-1}, t\right)}-1\right. \\
& \left.+\frac{1}{M_{F}\left(w_{i}, G w_{i}, t\right)}-1+\frac{1}{M_{F}\left(w_{i}, G w_{i-1}, t\right)}-1+\frac{1}{M_{F}\left(w_{i-1}, G w_{i}, t\right)}-1\right) \\
& +c\left(\frac{1}{\min \left\{M_{F}\left(w_{i-1}, G w_{i-1}, t\right), M_{F}\left(w_{i}, G w_{i}, t\right), M_{F}\left(w_{i}, G w_{i-1}, t\right), M_{F}\left(w_{i-1}, G w_{i}, t\right)\right\}}-1\right) \\
\leq & a\left(\frac{1}{M_{F}\left(w_{i-1}, w_{i}, t\right)}-1\right)+b\left(\frac{1}{M_{F}\left(w_{i-1}, w_{i}, t\right)}-1+\frac{1}{M_{F}\left(w_{i}, w_{i+1}, t\right)}-1+\frac{1}{M_{F}\left(w_{i-1}, w_{i}, t\right)}-1\right. \\
& \left.+\frac{1}{M_{F}\left(w_{i}, w_{i+1}, t\right)}-1\right)+c\left(\frac{1}{\min \left\{M_{F}\left(w_{i-1}, w_{i}, t\right), M_{F}\left(w_{i-1}, w_{i}, t\right), 1, M_{F}\left(w_{i}, w_{i+1}, t\right)\right\}}-1\right) \\
= & a\left(\frac{1}{M_{F}\left(w_{i-1}, w_{i}, t\right)}-1\right)+b\left(\frac{1}{M_{F}\left(w_{i-1}, w_{i}, t\right)}-1+\frac{1}{M_{F}\left(w_{i}, w_{i+1}, t\right)}-1+\frac{1}{M_{F}\left(w_{i-1}, w_{i}, t\right)}-1\right. \\
& \left.+\frac{1}{M_{F}\left(w_{i}, w_{i+1}, t\right)}-1\right)+c\left(\frac{1}{\min \left\{M_{F}\left(w_{i-1}, w_{i}, t\right), M_{F}\left(w_{i-1}, w_{i}, t\right), M_{F}\left(w_{i}, w_{i+1}, t\right)\right\}}-1\right) .
\end{aligned}
$$


Three possibilities arise:

$$
\begin{aligned}
\frac{1}{M_{F}\left(w_{i}, w_{i+1}, t\right)}-1 \leq & a\left(\frac{1}{M_{F}\left(w_{i-1}, w_{i}, t\right)}-1\right)+2 b\left(\frac{1}{M_{F}\left(w_{i-1}, w_{i}, t\right)}-1+\frac{1}{M_{F}\left(w_{i}, w_{i+1}, t\right)}-1\right) \\
& +c\left(\frac{1}{\min \left\{M_{F}\left(w_{i-1}, w_{i}, t\right), M_{F}\left(w_{i}, w_{i+1}, t\right), M_{F}\left(w_{i-1}, w_{i+1}, t\right)\right\}}-1\right) .
\end{aligned}
$$

(i) If $M_{F}\left(w_{i-1}, w_{i}, t\right)$ is the minimum term in $\left\{M_{F}(\right.$ $\left.\left.w_{i-1}, w_{i}, t\right), M_{F}\left(w_{i}, w_{i+1}, t\right), M_{F}\left(w_{i-1}, w_{i+1}, t\right)\right\}$, then, after simplification, (10) can be written as

$$
\begin{aligned}
& \frac{1}{M_{F}\left(w_{i}, w_{i+1}, t\right)}-1 \\
& \quad \leq \frac{a+2 b+c}{1-2 b}\left(\frac{1}{M_{F}\left(w_{i-1}, w_{i}, t\right)}-1\right), \quad \text { for } t>0
\end{aligned}
$$

(ii) If $M_{F}\left(w_{i}, w_{i+1}, t\right)$ is the minimum term in $\left\{M_{F}(\right.$ $\left.\left.w_{i-1}, w_{i}, t\right), M_{F}\left(w_{i}, w_{i+1}, t\right), M_{F}\left(w_{i-1}, w_{i+1}, t\right)\right\}$, then again, (10) can be written as

$$
\begin{aligned}
& \frac{1}{M_{F}\left(w_{i}, w_{i+1}, t\right)-1} \\
& \quad \leq \frac{a+2 b}{1-2 b-c}\left(\frac{1}{M_{F}\left(w_{i-1}, w_{i}, t\right)}-1\right), \quad \text { for } t>0
\end{aligned}
$$

(iii) If $M_{F}\left(w_{i-1}, w_{i+1}, t\right)$ is the minimum term in $\left\{M_{F}(\right.$ $\left.\left.w_{i-1}, w_{i}, t\right), M_{F}\left(w_{i}, w_{i+1}, t\right), M_{F}\left(w_{i-1}, w_{i+1}, t\right)\right\}$, then again, (10) becomes

$$
\begin{aligned}
& \frac{1}{M_{F}\left(w_{i}, w_{i+1}, t\right)}-1 \\
& \quad \leq \frac{a+2 b+c}{1-2 b-c}\left(\frac{1}{M_{F}\left(w_{i-1}, w_{i}, t\right)}-1\right), \quad \text { for } t>0
\end{aligned}
$$

Let $\beta:=\max \{(a+2 b+c) /(1-2 b),(a+2 b) /(1-2 b-c)$ $,(a+2 b+c) /(1-2 b-c)\}<1$. Then, from all cases, we get

$$
\frac{1}{M_{F}\left(w_{i}, w_{i+1}, t\right)}-1 \leq \beta\left(\frac{1}{M_{F}\left(w_{i-1}, w_{i}, t\right)}-1\right), \quad \text { for } t>0
$$

Similarly,

$$
\frac{1}{M_{F}\left(w_{i-1}, w_{i}, t\right)}-1 \leq \beta\left(\frac{1}{M_{F}\left(w_{i-2}, w_{i-1}, t\right)}-1\right), \quad \text { for } t>0
$$

Now, from (14) and (15) and by induction, for $t>0$,

$$
\begin{aligned}
& \frac{1}{M_{F}\left(w_{i}, w_{i+1}, t\right)}-1 \\
& \quad \leq \beta\left(\frac{1}{M_{F}\left(w_{i-1}, w_{i}, t\right)}-1\right) \leq \beta^{2}\left(\frac{1}{M_{F}\left(w_{i-2}, w_{i-1}, t\right)}-1\right) \\
& \quad \leq \cdots \leq \beta^{i}\left(\frac{1}{M_{F}\left(w_{0}, w_{1}, t\right)}-1\right) \longrightarrow 0, \quad \text { as } i \longrightarrow \infty .
\end{aligned}
$$

This yields that

$$
\lim _{i \longrightarrow \infty} M_{F}\left(w_{i}, w_{i+1}, t\right)=1, \quad \text { for } t>0 \text {. }
$$

Since $M_{F}$ is triangular, we have

$$
\begin{aligned}
\frac{1}{M_{F}\left(w_{i}, w_{k}, t\right)}-1 \\
\leq\left(\frac{1}{M_{F}\left(w_{i}, w_{i+1}, t\right)}-1\right)+\left(\frac{1}{M_{F}\left(w_{i+1}, w_{i+2}, t\right)}-1\right) \\
\quad+\cdots+\left(\frac{1}{M_{F}\left(w_{k-1}, w_{k}, t\right)}-1\right) \\
\leq\left(\beta^{i}+\beta^{i+1}+\cdots+\beta^{k-1}\right)\left(\frac{1}{M_{F}\left(w_{0}, w_{1}, t\right)}-1\right) \\
\leq \quad\left(\frac{\beta^{i}}{1-\beta}\right)\left(\frac{1}{M_{F}\left(w_{0}, w_{1}, t\right)}-1\right) \longrightarrow 0, \quad \text { as } i \longrightarrow \infty
\end{aligned}
$$

Thus, $\left\{w_{i}\right\}$ is a Cauchy sequence. Since $W$ is complete, 
there is $\kappa \in W$ so that

$$
\lim _{i \longrightarrow \infty} M_{F}\left(\kappa, w_{i}, t\right)=1, \quad \text { for } t>0 .
$$

$$
\begin{aligned}
\frac{1}{M_{F}(\kappa, G \kappa, t)}-1 \leq & \left.\frac{1}{M_{F}\left(\kappa, w_{i+1}, t\right)}-1\right) \\
& +\left(\frac{1}{M_{F}\left(w_{i+1}, G \kappa, t\right)}-1\right), \quad \text { for } t>0 .
\end{aligned}
$$

We shall show that $G \kappa=\kappa$. By the triangular property of $M_{F}$, we have that
Now, by using (8), (17), and (19), we have

$$
\begin{aligned}
\frac{1}{M_{F}\left(w_{i+1}, G \kappa, t\right)}-1 \leq & \frac{1}{M_{F}\left(G w_{i}, G \kappa, t\right)}-1 \leq a\left(\frac{1}{M_{F}\left(w_{i}, \kappa, t\right)}-1\right)+b\left(\frac{1}{M_{F}\left(w_{i}, G w_{i}, t\right)}-1+\frac{1}{M_{F}(\kappa, G \kappa, t)}-1 \frac{1}{M_{F}\left(\kappa, G w_{i}, t\right)}\right. \\
& \left.-1+\frac{1}{M_{F}\left(w_{i}, G \kappa, t\right)}-1\right)+c\left(\frac{1}{\min \left\{M_{F}\left(w_{i}, G w_{i}, t\right), M_{F}(\kappa, G \kappa, t), M_{F}\left(\kappa, G w_{i}, t\right), M_{F}\left(w_{i}, G \kappa, t\right)\right\}}-1\right) \\
= & a\left(\frac{1}{M_{F}\left(w_{i}, \kappa, t\right)}-1\right)+b\left(\frac{1}{M_{F}\left(w_{i}, w_{i+1}, t\right)}-1+\frac{1}{M_{F}(\kappa, G \kappa, t)}-1 \frac{1}{M_{F}\left(\kappa, w_{i+1}, t\right)}-1\right. \\
& \left.+\frac{1}{M_{F}\left(w_{i}, G \kappa, t\right)}-1\right)+c\left(\frac{1}{\min \left\{M_{F}\left(w_{i}, G w_{i+1}, t\right), M_{F}(\kappa, G \kappa, t), M_{F}\left(\kappa, w_{i+1}, t\right), M_{F}\left(w_{i}, G \kappa, t\right)\right\}}-1\right) \\
\longrightarrow & 2 b\left(\frac{1}{M_{F}(\kappa, G \kappa, t)}-1\right)+c\left(\frac{1}{\min \left\{1, M_{F}(\kappa, G \kappa, t)\right\}}-1\right), \quad \text { as } i \longrightarrow \infty .
\end{aligned}
$$

Hence,

$$
\begin{aligned}
& \lim _{i \longrightarrow \infty} \sup \frac{1}{M_{F}\left(w_{i+1}, G \kappa, t\right)}-1 \\
& \quad \leq(2 b+c)\left(\frac{1}{M_{F}(\kappa, G \kappa, t)}-1\right), \quad \text { for } t>0 .
\end{aligned}
$$

Equation (22) together with (20) and (19) implies that

$$
\frac{1}{M_{F}(\kappa, G \kappa, t)}-1 \leq(2 b+c)\left(\frac{1}{M_{F}(\kappa, G \kappa, t)}-1\right), \quad \text { for } t>0
$$

As $(2 b+c)<1$, one has $M_{F}(\kappa, G \kappa, t)=1$. This implies that $G \kappa=\kappa$.

The uniqueness is as follows: let $\kappa^{*} \in W$ be such that $G$ $\kappa^{*}=\kappa^{*}$. Then, in view of (8), we have for $t>0$

$$
\begin{aligned}
\frac{1}{M_{F}\left(\kappa, \kappa^{*}, t\right)}-1 \leq & a\left(\frac{1}{M_{F}\left(\kappa, \kappa^{*}, t\right)}-1\right)+b\left(\frac{1}{M_{F}\left(\kappa, G \kappa_{i}, t\right)}-1+\frac{1}{M_{F}\left(\kappa^{*}, G \kappa^{*}, t\right)}-1 \frac{1}{M_{F}\left(\kappa, G \kappa^{*}, t\right)}-1+\frac{1}{M_{F}\left(\kappa^{*}, G \kappa, t\right)}-1\right) \\
& +c\left(\frac{1}{\min \left\{M_{F}\left(\kappa, G \kappa^{*}, t\right), M_{F}\left(\kappa^{*}, G \kappa^{*}, t\right), M_{F}\left(\kappa, G \kappa_{i}^{*}, t\right), M_{F}\left(\kappa^{*}, G \kappa, t\right)\right\}}-1\right) \\
= & a\left(\frac{1}{M_{F}\left(\kappa, \kappa^{*}, t\right)}-1\right)+2 b\left(\frac{1}{M_{F}\left(\kappa, G \kappa^{*}, t\right)}-1\right)+c\left(\frac{1}{\min \left\{1, M_{F}\left(\kappa, \kappa^{*}, t\right)\right\}}-1\right) \\
= & (a+2 b+c)\left(\frac{1}{M_{F}\left(\kappa, \kappa^{*}, t\right)}-1\right) \leq(a+2 b+c)\left(\frac{1}{M_{F}\left(G \kappa, G \kappa^{*}, t\right)}-1\right) \\
\leq & (a+2 b+c)^{2}\left(\frac{1}{M_{F}\left(\kappa, \kappa^{*}, t\right)}-1\right) \leq \cdots \leq(a+2 b+c)^{i}\left(\frac{1}{M_{F}\left(\kappa, \kappa^{*}, t\right)}-1\right) \longrightarrow 0, \quad \text { as } i \longrightarrow \infty .
\end{aligned}
$$


Hence, we get that $M_{F}\left(\kappa, \kappa^{*}, t\right)=1$, so $\kappa=\kappa^{*}$. Thus, $G$ has a unique fixed point in $W$.

Corollary 9. Let $\left(W, M_{F}, *\right)$ be a complete FM-space so that the fuzzy metric $M_{F}$ is triangular. Let $G: W \longrightarrow W$ verify that

$$
\begin{aligned}
\frac{1}{M_{F}(G w, G x, t)}-1 \leq & a\left(\frac{1}{M_{F}(w, x, t)}-1\right)+b\left(\frac{1}{M_{F}(w, G w, t)}\right. \\
& -1+\frac{1}{M_{F}(x, G x, t)}-1+\frac{1}{M_{F}(x, G w, t)} \\
& \left.-1+\frac{1}{M_{F}(w, G x, t)}-1\right)
\end{aligned}
$$

for all $w, x \in W, t>0, a \in(0,1)$, and $b \in[0,1 / 4)$ with $(a+$ $2 b)<1$. Then, $G$ has a unique fixed point.

Proof. It follows by putting $c=0$ in Theorem (8).

Example 10. Let $W=[0,1]$ be equipped with a continuous $t$ -norm. Let $M_{F}: W \times W \times(0, \infty) \longrightarrow[0,1]$ be a fuzzy metric defined by

$$
M_{F}(w, x, t)=\frac{t}{t+d(w, x)}, \quad \text { where } d(w, x)=\left|\frac{w-x}{3}\right| \text {, }
$$

for all $w, x \in W$ and $t>0$. Then, $\left(W, M_{F}, *\right)$ is a complete FM-space. Now, we define the mapping $G: W \longrightarrow W$ by

$$
G(w)=\frac{2 w}{3}+\frac{4}{15}, \quad \text { for all } w \in[0,1] \text { and } t>0
$$

Then,

$$
\begin{aligned}
\frac{1}{M_{F}(G w, G x, t)}-1 \\
=\left|\frac{G w-G x}{3 t}\right|=\left|\frac{2(w-x)}{9 t}\right| \leq\left|\frac{2(w-x)}{9 t}\right| \\
+\frac{1}{15}\left|\frac{2(5 w+5 x-8)}{45 t}\right|=\frac{2}{3}\left|\frac{w-x}{3 t}\right| \\
+\frac{1}{15}||+\frac{1}{3 t}\left(\frac{15 x-10 w-4}{15}\right)|+| \frac{1}{3 t}\left(\frac{15 w-10 x-4}{15}\right) \mid \\
=\frac{2}{3}\left|\frac{w-x}{3 t}\right|+\frac{1}{15}\left(\left|\frac{1}{3 t}\left(w-\frac{2 w}{3}-\frac{4}{15}\right)\right|+\left|\frac{1}{3 t}\left(\frac{5 x-4}{15}\right)\right|\right. \\
+\left|\frac{1}{3 t}\left(x-\frac{2 w x}{3}-\frac{4}{15}\right)\right|+\left|\frac{1}{3 t}\left(x-\frac{2 w}{3}-\frac{4}{15}\right)\right| \\
\left.+\left|\frac{1}{3 t}\left(w-\frac{2 x}{3}-\frac{4}{15}\right)\right|\right)=\frac{2}{3}\left(\frac{1}{M_{F}(w, x, t)}-1\right) \\
+\frac{1}{15}\left(\frac{1}{M_{F}(w, G w, t)}-1+\frac{1}{M_{F}(x, G x, t)}-1\right. \\
\left.+\frac{1}{M_{F}(x, G w, t)}-1+\frac{1}{M_{F}(w, G x, t)}-1\right) .
\end{aligned}
$$

Hence, all the conditions of Corollary 9 are satisfied with $a=2 / 3$ and $b=1 / 15$. Hence, the self-mapping $G$ has a unique fixed point, that is, $G(4 / 5)=4 / 5 \in[0,1]$.

Corollary 11. Let $\left(W, M_{F}, *\right)$ be a complete FM-space so that the fuzzy metric $M_{F}$ is triangular. Let $G: W \longrightarrow W$ verify that

$$
\frac{1}{M_{F}(G w, G x, t)}-1 \leq a\left(\frac{1}{M_{F}(w, x, t)}-1\right)+c\left(\frac{1}{\min \left\{M_{F}(w, G w, t), M_{F}(x, G x, t), M_{F}(x, G w, t), M(w, G x, t)\right\}}-1\right)
$$

for all $w, x \in W, t>0, a \in(0,1)$, and $c \in[0,1)$ with $(a+2 c)$ $<1$. Then, $G$ has a unique fixed point.

Proof. It follows by putting $b=0$ in Theorem (8).

Example 12. Let $W=[0, \infty)$ be endowed with a continuous $t$ -norm. Let $M_{F}: W \times W \times(0, \infty) \longrightarrow[0,1]$ be a fuzzy metric defined by

$$
M_{F}(w, x, t)=\frac{t}{t+d(w, x)} \text { where } d(w, x)=|w-x|
$$

for all $w, x \in W$ and $t>0$. Then, $\left(W, M_{F}, *\right)$ is a complete FM-space. Now, we define a mapping $G: W \longrightarrow W$ by

$$
G w=\left(\begin{array}{ll}
\frac{3 w}{4}+\frac{1}{2}, & \text { if } w \in[0,1] \\
\frac{w}{2}+\frac{7}{2}, & \text { if } w \in(1, \infty)
\end{array}\right.
$$

We have

$$
\begin{aligned}
\frac{1}{M_{F}(G w, G x, t)}-1= & \frac{3}{4 t}|w-x|=\frac{3}{4 t}\left(\frac{1}{M_{F}(w, x, t)}-1\right) \leq \frac{3}{4}\left(\frac{1}{M_{F}(w, x, t)}-1\right) \\
& +\frac{1}{9}\left(\frac{1}{\min \left\{M_{F}(w, G w, t), M(x, G x, t), M_{F}(x, G w, t), M_{F}(w, G x, t)\right\}}-1\right) .
\end{aligned}
$$


Hence, all the conditions of Corollary 11 are satisfied with $a=3 / 4$ and $b=1 / 9$. Then, the self-mapping $G$ has a unique fixed point, that is, $G(7)=7$.

\section{4. Ćirić-Type Fuzzy Contraction Results in FM- Spaces}

In this section, we define Ćirić-type fuzzy contraction mappings and we present a unique related fixed-point theorem on a complete FM-space.

Definition 13. Let $\left(W, M_{F}, *\right)$ be a complete FM-space. A self-mapping $G: W \longrightarrow W$ is said to be a Cirić contraction if there is $\alpha \in(0,1)$ such that

$$
\begin{aligned}
& \frac{1}{M_{F}(G w, G x, t)}-1 \\
& \leq \alpha \max \left\{\begin{array}{c}
\frac{1}{M_{F}(w, G w, t)}-1, \frac{1}{M_{F}(x, G x, t)}-1 \\
\frac{1}{M_{F}(x, G w, t)}-1, \frac{1}{M_{F}(w, G x, t)}-1 \\
\frac{1}{M_{F}(w, x, t)}-1
\end{array}\right\},
\end{aligned}
$$

for all $w, x \in W$ and $t>0$. Here, $\alpha$ is called the contractive constant of $T$.

Theorem 14. Let a self-mapping $G: W \longrightarrow W$ be a Cirić contraction in a complete FM-space $\left(W, M_{F}, *\right)$ so that $M_{F}$ is triangular and (33) satisfies with $2 \alpha<1$. Then, $G$ has a unique fixed point.

Proof. Fix $w_{0} \in W$. Take an iterative sequence $\left\{w_{i}\right\}$ such that $w_{i+1}=G w_{i}$ for all $i \geq 0$. Now, by using (33), we have

$$
\begin{aligned}
& \frac{1}{M_{F}\left(w_{i}, w_{i+1}, t\right)}-1 \\
& =\frac{1}{M_{F}\left(G w_{i-1}, G w_{i}, t\right)}-1 \\
& \quad \leq \alpha \max \left\{\begin{array}{c}
\frac{1}{M_{F}\left(w_{i-1}, G w_{i-1}, t\right)}-1, \frac{1}{M_{F}\left(w_{i}, G w_{i}, t\right)}-1 \\
\frac{1}{M_{F}\left(w_{i-1}, G w_{i}, t\right)}-1, \frac{1}{M_{F}\left(w_{i}, G w_{i-1}, t\right)}-1 \\
\frac{1}{M_{F}\left(w_{i-1}, w_{i}, t\right)}-1
\end{array}\right\}
\end{aligned}
$$

After simplification, for $t>0$, we get

$$
\begin{aligned}
& \frac{1}{M_{F}\left(w_{i}, w_{i+1}, t\right)}-1 \\
& \quad \leq \alpha \max \left\{\frac{1}{M_{F}\left(w_{i-1}, w_{i}, t\right)}-1, \frac{1}{M_{F}\left(w_{i}, w_{i+1}, t\right)}\right. \\
& \left.\quad-1, \frac{1}{M_{F}\left(w_{i-1}, w_{i+1}, t\right)}-1\right\} .
\end{aligned}
$$

Now, there are three possibilities:

(i) If $\left(1 / M_{F}\left(w_{i-1}, w_{i}, t\right)\right)-1$ is the maximum in $\{(1 /$ $\left.M_{F}\left(w_{i-1}, w_{i}, t\right)\right)-1,\left(1 / M_{F}\left(w_{i}, w_{i+1}, t\right)\right)-1,\left(1 / M_{F}(\right.$ $\left.\left.\left.w_{i-1}, w_{i+1}, t\right)\right)-1\right\}$, then, from (35), we have

$$
\frac{1}{M_{F}\left(w_{i}, w_{i+1}, t\right)}-1 \leq \alpha\left(\frac{1}{M_{F}\left(w_{i-1}, w_{i}, t\right)}-1\right), \quad \text { for } t>0
$$

(ii) If $\left(1 / M_{F}\left(w_{i}, w_{i+1}, t\right)\right)-1$ is the maximum in $\{(1 /$ $\left.M_{F}\left(w_{i-1}, w_{i}, t\right)\right)-1,\left(1 / M_{F}\left(w_{i}, w_{i+1}, t\right)\right)-1,\left(1 / M_{F}\right.$ $\left.\left.\left(w_{i-1}, w_{i+1}, t\right)\right)-1\right\}$, then, from (35), we have

$$
\frac{1}{M_{F}\left(w_{i}, w_{i+1}, t\right)}-1 \leq \alpha\left(\frac{1}{M_{F}\left(w_{i}, w_{i+1}, t\right)}-1\right),
$$

$$
\text { for } t>0 \text {, which is not possible }
$$

(iii) If $\left(1 / M_{F}\left(w_{i-1}, w_{i+1}, t\right)\right)-1$ is the maximum in $\{(1 /$ $\left.M_{F}\left(w_{i-1}, w_{i}, t\right)\right)-1,\left(1 / M_{F}\left(w_{\mathrm{i}}, w_{i+1}, t\right)\right)-1,\left(1 / M_{F}(\right.$ $\left.\left.\left.w_{i-1}, w_{i+1}, t\right)\right)-1\right\}$, then, from (35), we have

$$
\begin{aligned}
& \frac{1}{M_{F}\left(w_{i}, w_{i+1}, t\right)}-1 \\
& \quad \leq \alpha\left(\frac{1}{M_{F}\left(w_{i-1}, w_{i+1}, t\right)}-1\right) \\
& \quad \leq \frac{\alpha}{1-\alpha}\left(\frac{1}{M_{F}\left(w_{i-1}, w_{i}, t\right)}-1\right), \quad \text { for } t>0
\end{aligned}
$$

Let $\delta:=\max \{\alpha, a /(1-\alpha)\}<1$. Using (36) and (38), we have

$$
\frac{1}{M_{F}\left(w_{i}, w_{i+1}, t\right)}-1 \leq \delta\left(\frac{1}{M_{F}\left(w_{i-1}, w_{i}, t\right)}-1\right), \quad \text { for } t>0 .
$$


Similarly,

$\frac{1}{M_{F}\left(w_{i-1}, w_{i}, t\right)}-1 \leq \delta^{2}\left(\frac{1}{M_{F}\left(w_{i-2}, w_{i-1}, t\right)}-1\right), \quad$ for $t>0$.

Now, from (39) and (40) and by induction, for $t>0$

$$
\begin{aligned}
& \frac{1}{M_{F}\left(w_{i}, w_{i+1}, t\right)}-1 \\
& \quad \leq \delta\left(\frac{1}{M_{F}\left(w_{i-1}, w_{i}, t\right)}-1\right) \leq \delta^{2}\left(\frac{1}{M_{F}\left(w_{i-2}, w_{i-1}, t\right)}-1\right) \\
& \quad \leq \cdots \leq \delta^{i}\left(\frac{1}{M_{F}\left(w_{0}, w_{1}, t\right)}-1\right) \longrightarrow 0, \quad \text { as } i \longrightarrow \infty
\end{aligned}
$$

This yields that

$$
\lim _{i \longrightarrow \infty} M_{F}\left(w_{i}, w_{i+1}, t\right)=1, \quad \text { for } t>0 .
$$

Since $M_{F}$ is triangular and $k>i$, we have

$$
\begin{aligned}
& \frac{1}{M_{F}\left(w_{i}, w_{k}, t\right)}-1 \\
& \quad \leq\left(\frac{1}{M_{F}\left(w_{i}, w_{i+1}, t\right)}-1\right)+\left(\frac{1}{M_{F}\left(w_{i+1}, w_{i+2}, t\right)}-1\right)+\cdots \\
& \quad+\left(\frac{1}{M_{F}\left(w_{k-1}, w_{k}, t\right)}-1\right) \leq\left(\delta^{i}+\delta^{i+1}+\cdots+\delta^{k-1}\right) \\
& \quad \cdot\left(\frac{1}{M_{F}\left(w_{0}, w_{1}, t\right)}-1\right) \leq\left(\frac{\delta^{i}}{1-\delta}\right)\left(\frac{1}{M_{F}\left(w_{0}, w_{1}, t\right)}-1\right) \\
& \quad \longrightarrow 0, \quad \text { as } i \longrightarrow \infty .
\end{aligned}
$$

Hence, $\left\{w_{i}\right\}$ is a Cauchy sequence. Since $\left(W, M_{F}, *\right)$ is complete, there is $\kappa \in W$ so that

$$
\lim _{i \longrightarrow \infty} M_{F}\left(\kappa, w_{i}, t\right)=1, \quad \text { for } t>0 .
$$

Now, we have to show that $G \kappa=\kappa$. Since $M_{F}$ is triangular, one writes

$$
\begin{aligned}
\frac{1}{M_{F}(G \kappa, \kappa, t)}-1 \leq & \left(\frac{1}{M_{F}\left(G \kappa, w_{i+1}, t\right)}-1\right) \\
& +\left(\frac{1}{M_{F}\left(w_{i+1}, \kappa, t\right)}-1\right), \quad \text { for } t>0 .
\end{aligned}
$$

In view of (33), (42), and (44), we have for $t>0$

$$
\begin{aligned}
& \frac{1}{M_{F}\left(w_{i+1}, G \kappa, t\right)}-1 \\
& \leq \frac{1}{M_{F}\left(G w_{i}, G \kappa, t\right)}-1 \\
& \leq \alpha \max \left\{\begin{array}{l}
\frac{1}{M_{F}\left(w_{i}, G w_{i}, t\right)}-1, \frac{1}{M_{F}(\kappa, G \kappa, t)}-1 \\
\frac{1}{M_{F}\left(w_{i}, G \kappa, t\right)}-1, \frac{1}{M\left(\kappa, G w_{i}, t\right)}-1 \\
\frac{1}{M_{F}\left(w_{i}, \kappa, t\right)}-1
\end{array}\right\} \\
& \quad \alpha \max \left\{\begin{array}{c}
\frac{1}{M_{F}\left(w_{i}, w_{i+1}, t\right)}-1, \frac{1}{M_{F}(\kappa, G \kappa, t)}-1 \\
\frac{1}{M_{F}\left(w_{i}, G \kappa, t\right)}-1, \frac{1}{M_{F}\left(\kappa, w_{i+1}, t\right)}-1 \\
\frac{1}{M_{F}\left(w_{i}, \kappa, t\right)}-1
\end{array}\right\}
\end{aligned}
$$

Hence,

$$
\begin{aligned}
& \lim _{i \longrightarrow \infty} \sup \frac{1}{M_{F}\left(w_{i+1}, G \kappa, t\right)}-1 \\
& \quad \leq \alpha\left(\frac{1}{M_{F}(\kappa, G \kappa, t)}-1\right), \quad \text { for } t>0 .
\end{aligned}
$$

This together with (45) and (44), we have

$$
\frac{1}{M_{F}(\kappa, G \kappa, t)}-1 \leq \alpha\left(\frac{1}{M_{F}(\kappa, G \kappa, t)}-1\right), \quad \text { for } t>0 .
$$

Since $\alpha \in(0,1)$, one gets $M_{F}(\kappa, G \kappa, t)=1$. This implies that $G \kappa=\kappa$.

The uniqueness is as follows: let $\kappa^{*} \in W$ be such that $G$ $\kappa=\kappa^{*}$. Using (33), we have

$$
\begin{aligned}
& \frac{1}{M_{F}\left(G \kappa, G \kappa^{*}, t\right)}-1 \\
& \leq \alpha \max \left\{\begin{array}{c}
\frac{1}{M_{F}(\kappa, G \kappa, t)}-1, \frac{1}{M_{F}\left(\kappa^{*}, G \kappa^{*}, t\right)}-1 \\
\frac{1}{M_{F}\left(\kappa, G \kappa^{*}, t\right)}-1, \frac{1}{M_{F}\left(\kappa^{*}, G \kappa, t\right)}-1 \\
\frac{1}{M_{F}\left(\kappa, \kappa^{*}, t\right)}-1
\end{array}\right\}
\end{aligned}
$$




$$
\begin{aligned}
& =\alpha\left(\frac{1}{M_{F}\left(\kappa, \kappa^{*}, t\right)}-1\right) \leq \alpha\left(\frac{1}{M_{F}\left(G \kappa, G \kappa^{*}, t\right)}-1\right) \\
& \leq \alpha^{2}\left(\frac{1}{M_{F}\left(\kappa, \kappa^{*}, t\right)}-1\right) \leq \cdots \\
& \leq \alpha^{i}\left(\frac{1}{M_{F}\left(\kappa, \kappa^{*}, t\right)}-1\right) \longrightarrow 0, \quad \text { as } i \longrightarrow \infty .
\end{aligned}
$$

We get that $M_{F}\left(\kappa, \kappa^{*}, t\right)=1$ for $t>0$. This implies that $\kappa=\kappa^{*}$. Thus, $G$ has a unique fixed point in $W$.

Example 15. Let $W=[0, \infty)$ be endowed with a continuous $t$ -norm. Let a fuzzy metric $M_{F}: W \times W \times(0, \infty) \longrightarrow[0,1]$ be defined by

$$
M_{F}(w, x, t)=\frac{t}{t+d(w, x)}, \quad \text { where } d(w, x)=2|w-x|
$$

for all $w, x \in W$ and $t>0$. Then, $\left(W, M_{F}, *\right)$ is a complete FM-space. Now, we define a mapping $G: W \longrightarrow W$ as

$$
G w= \begin{cases}\frac{w}{3}+\frac{1}{2}, & \text { if } w \in[0,1] \\ \frac{7 w}{8}+\frac{2}{3}, & \text { if } w \in(1, \infty)\end{cases}
$$

We have

$$
\begin{aligned}
& \frac{1}{M_{F}(G w, G x, t)}-1 \\
& =\frac{2}{t}\left|\frac{w}{3}-\frac{x}{3}\right|=\frac{1}{3}\left(\frac{1}{M(w, x, t)}-1\right) \\
& \leq \frac{1}{3} \max \left(\begin{array}{c}
\frac{1}{M_{F}(w, G w, t)}-1, \frac{1}{M_{F}(x, G x, t)}-1 \\
\frac{1}{M_{F}(x, G w, t)}-1, \frac{1}{M_{F}(w, G \mathrm{x}, t)}-1 \\
\frac{1}{M_{F}(w, x, t)}-1
\end{array}\right),
\end{aligned}
$$

for $t>0$. Hence, all the conditions of Theorem 14 are satisfied with $\alpha=1 / 3$ and $G$ has a unique fixed point, that is, $G$ $(16 / 3)=16 / 3$.

\section{Application}

In this section, we present an integral-type equation. Let $W=C([0, \xi], \mathbb{R})$ be the space of all real-valued continuous functions on the interval $[0, \xi]$, where $0<\xi \in \mathbb{R}$. Now, we present a particular case of a Fredholm integral equation
(FIE) of the second kind given as follows:

$$
w(\tau)=\int_{0}^{\xi} K(\tau, v, w(v)) d v
$$

where $\tau \in[0, \xi]$ and $K:[0, \xi] \times[0, \xi] \times \mathbb{R} \longrightarrow \mathbb{R}$. The induced metric $d: W \times W \longrightarrow \mathbb{R}$ is defined by

$$
d(w, x)=\|w-x\|, \quad \forall w, x \in W
$$

The binary operation $*$, being a continuous $t$-norm, is defined by $\alpha * \beta=\alpha \beta$ for all $\alpha, \beta \in[0, \xi]$. The standard fuzzy metric $M_{F}: W \times W \times(0, \infty) \longrightarrow[0,1]$ can be expressed as

$$
M_{F}(w, x, t)=\frac{t}{t+d(w, x)}, \quad \forall w, x \in W \text {, and } t>0
$$

Then easily, we can show that $M_{F}$ is triangular and ( $W$ , $\left.M_{F}, *\right)$ is a complete $F M-$ space.

Theorem 16. Assume that there is $\eta \in(0,1)$ so that

$$
\|G w-G x\| \leq \eta N(G, w, x), \quad \forall w, x \in W
$$

where

$$
\begin{aligned}
& N(G, w, x) \\
& \quad=\max \left\{\begin{array}{c}
\|w-x\|,\|G w-w\|,\|G x-x\|,\|G w-x\|,\|G x-w\| \\
\|G w-w\|+\|G x-x\|+\|G w-x\|+\|G x-w\|,
\end{array}\right\} .
\end{aligned}
$$

Then, the FIE (53) has a unique solution.

Proof. Give $G: W \longrightarrow W$ as

$$
G w(\tau)=\int_{0}^{\xi} K(\tau, v, w(v)) d v
$$

Notice that $G$ is well defined and (53) has a unique solution if and only if $G$ has a unique fixed point in $W$. Now, we have to show that Theorem 8 is applied to the integral operator $G$. Then, for all $w, x \in W$, we have the following six cases:

(1) If $\|w-x\|$ is the maximum term in (57), then $N(G$ , $w, x)=\|w-x\|$. Therefore, in view of (55) and (56), we have

$$
\begin{aligned}
\frac{1}{M_{F}(G w, G x, t)}-1 & =\frac{d(G w, G x)}{t} \leq \eta \frac{N(G, w, x)}{t} \\
& =\eta \frac{\|w-x\|}{t}=\eta\left(\frac{1}{M_{F}(w, x, t)}-1\right) .
\end{aligned}
$$


This implies that

$$
\frac{1}{M_{F}(G w, G x, t)}-1 \leq \eta\left(\frac{1}{M_{F}(w, x, t)}-1\right), \quad \text { for } t>0,
$$

for all $w, x \in W$ such that $G w \neq G x$. The inequality (60) holds if $G w=G x$. Thus, the integral operator $G$ satisfies all the conditions of Theorem 8 with $\eta=a$ and $b=c=0$ in (8). Then, the integral operator $G$ has a unique fixed point, i.e., (53) has a solution in $W$

(2) If $\|G w-w\|$ is the maximum term in (57), then, $N$ $(G, w, x)=\|G w-w\|$. Therefore, using (55) and (56), we have

$$
\begin{aligned}
\frac{1}{M_{F}(G w, G x, t)}-1 & =\frac{d(G w, G x)}{t} \leq \eta \frac{N(G, w, x)}{t} \\
& =\eta \frac{\|G w-w\|}{t}=\eta\left(\frac{1}{M_{F}(G w, w, t)}-1\right) .
\end{aligned}
$$

It yields that

$$
\frac{1}{M_{F}(G w, G x, t)}-1 \leq \eta\left(\frac{1}{M_{F}(G w, w, t)}-1\right), \quad \text { for } t>0,
$$

for all $w, x \in W$ such that $G w \neq G x$

(3) If $\|G x-x\|$ is the maximum term in (57), then $N($ $G, w, x)=\|G x-x\|$. Therefore, by (55) and (56), we have

$$
\begin{aligned}
\frac{1}{M_{F}(G w, G x, t)}-1 & =\frac{d(G w, G x)}{t} \leq \eta \frac{N(G, w, x)}{t} \\
& =\eta \frac{\|G x-x\|}{t}=\eta\left(\frac{1}{M(G x, x, t)}-1\right) .
\end{aligned}
$$

That is,

$$
\frac{1}{M_{F}(G w, G x, t)}-1 \leq \eta\left(\frac{1}{M_{F}(G x, x, t)}-1\right), \quad \text { for } t>0,
$$

for all $w, x \in W$ such that $G w \neq G x$

(4) If $\|G w-x\|$ is the maximum term in (57), then, $N(G, w, x)=\|G w-x\|$. Therefore, due to (55) and (56), we have

$$
\begin{aligned}
\frac{1}{M_{F}(G w, G x, t)}-1 & =\frac{d(G w, G x)}{t} \leq \eta \frac{N(G, w, x)}{t} \\
& =\eta \frac{\|G w-x\|}{t}=\eta\left(\frac{1}{M_{F}(G w, x, t)}-1\right) .
\end{aligned}
$$

Hence,

$$
\frac{1}{M_{F}(G w, G x, t)}-1 \leq \eta\left(\frac{1}{M_{F}(G w, x, t)}-1\right), \quad \text { for } t>0,
$$

for all $w, x \in W$ such that $G w \neq G x$

(5) If $\|G x-w\|$ is the maximum term in (57), then, $N($ $G, w, x)=\|G x-w\|$. Using (55) and (56), we have

$$
\begin{aligned}
\frac{1}{M_{F}(\mathrm{G} w, G x, t)}-1 & =\frac{d(G w, G x)}{t} \leq \eta \frac{N(G, w, x)}{t} \\
& =\eta \frac{\|G x-w\|}{t}=\eta\left(\frac{1}{M_{F}(G x, w, t)}-1\right) .
\end{aligned}
$$

It implies that

$$
\frac{1}{M_{F}(G w, G x, t)}-1 \leq \eta\left(\frac{1}{M_{F}(G x, w, t)}-1\right), \quad \text { for } t>0,
$$

for all $w, x \in W$ such that $G w \neq G x$

The inequalities (62), (64), (66), and (68) hold if $G w=$ $G x$. Thus, the integral operator $G$ satisfies all the conditions of Theorem 8 with $\eta=c$ and $a=b=0$ in (8). The integral operator $G$ has a unique fixed point, i.e., (53) has a solution in $W$.

(6) If $\|G w-w\|+\|G x-x\|+\|G w-x\|+\|G x-w\|$ is the maximum term in (57), then, $N(G, w, x)=\| G w$ $-w\|+\| G x-x\|+\| G w-x\|+\| G x-w \|$. Therefore, from (55) and (56), we have

$$
\begin{aligned}
\frac{1}{M_{F}(G w, G x, t)}-1 \\
=\frac{d(G w, G x)}{t} \leq \eta \frac{N(G, w, x)}{t} \\
=\eta \frac{\|G w-w\|+\|G x-x\|+\|G w-x\|+\|G x-w\|}{t} \\
=\eta\left(\frac{1}{M_{F}(w, G w, t)}-1+\frac{1}{M_{F}(x, G x, t)}-1\right. \\
\left.\quad+\frac{1}{M(x, G w, t)}-1+\frac{1}{M_{F}(w, G x, t)}-1\right) .
\end{aligned}
$$


That is,

$$
\begin{aligned}
& \frac{1}{M_{F}(G w, G x, t)}-1 \\
& \quad \leq \quad\left(\frac{1}{M_{F}(w, G w, t)}-1+\frac{1}{M_{F}(x, G x, t)}-1\right. \\
& \left.\quad+\frac{1}{M_{F}(x, G w, t)}-1+\frac{1}{M_{F}(w, G x, t)}-1\right), \quad \text { for } t>0,
\end{aligned}
$$

for all $w, x \in W$ such that $G w \neq G x$. The inequality (70) holds if $G w=G x$. Thus, the integral operator $G$ satisfies all the conditions of Theorem 8 with $\eta=b$ and $a=c=0$ in (8). The integral operator $G$ has a unique fixed point, i.e., (53) has a solution in $W$.

Now, we present a special type of example for a particular case of an FIE of a second kind.

Example 17. Take $W=[0,1]$. If we put $\xi=1$ in (53), then, we have the following integral equation:

$$
\begin{aligned}
w(\tau) & =\int_{0}^{1} \frac{2}{5(\tau+1+w(v))} d v, \quad \text { where } K(\tau, v, w(v)) \\
& =\frac{2}{5(\tau+1+w(v))}
\end{aligned}
$$

Equation (71) is a special kind of the integral equation (53), where $\tau \in[0,1]$. Then,

$$
\begin{aligned}
& \|K(\tau, v, w(v))-K(\tau, v, x(v))\| \\
& \quad=\left\|\frac{2}{5(\tau+1+w(v))}-\frac{2}{5(\tau+1+x(v))}\right\| \\
& \quad=\frac{2}{5}\left\|\frac{w(v)-x(v)}{(\tau+1+w(v))(\tau+1+x(v))}\right\| \\
& \quad \leq \frac{2}{5}\|w(v)-x(v)\|=\frac{2}{5} N(G, w, x),
\end{aligned}
$$

where $N(G, w, x)=\|w(v)-x(v)\|$. Now, we have to show that $\|G w(\tau)-G x(\tau)\| \leq \eta N(G, w, x)$. From equation (58), we have

$$
\begin{array}{rl}
\| G & w(\tau)-G x(\tau) \| \\
& =\left\|\int_{0}^{1} K(\tau, v, w(v)) d v-\int_{0}^{1} K(\tau, v, x(v)) d v\right\| \\
& =\int_{0}^{1}\|K(\tau, v, w(v))-K(\tau, v, x(v))\| d v \\
& \leq \int_{0}^{1} \frac{2}{5}\|w(v)-x(v)\| d v=\int_{0}^{1} \frac{2}{5} N(T, w, x) d v \\
& =\frac{2}{5} N(G, w, x) \int_{0}^{1} d v=\frac{2}{5} N(G, w, x) .
\end{array}
$$

Hence, all conditions of Theorem 16 hold with $\eta=2 / 5$ $<1$. The integral equation (71) has a unique solution by using Theorem 16.

\section{Conclusion}

In this paper, we proved variant unique fixed-point results for some generalized contraction-type self-mappings in complete FM-spaces, without continuity and by using the "triangular property of the fuzzy metric" as a basic tool. We presented illustrative examples. Moreover, we provided an application about a particular case of Fredholm integral equation of second kind. In this direction, researchers can prove more fixed-point results in complete FM-spaces without using continuity via different types of applications.

\section{Data Availability}

Data sharing is not applicable to this article as no dataset were generated or analysed during the current study.

\section{Conflicts of Interest}

The authors declare that they have no competing interests.

\section{Authors' Contributions}

All the authors have equally contributed to the final manuscript.

\section{References}

[1] S. Banach, "Sur les opérations dans les ensembles abstraits et leur application aux équations intégrales," Fundamenta Mathematicae, vol. 3, pp. 133-181, 1922.

[2] A. C. M. Ran and M. C. B. Reurings, "A fixed point theorem in partially ordered sets and some applications to matrix equations," Proceedings of American Mathematical Society, vol. 132, no. 5, pp. 1435-1443, 2004.

[3] J. J. Nieto and R. Rodríguez-López, "Contractive mapping theorems in partially ordered sets and applications to ordinary differential equations," Order, vol. 22, no. 3, pp. 223-239, 2005.

[4] J. J. Priskillal and P. Thangavelu, " $\$ \backslash p s i \$$-Contractive type fuzzy mapping and its applications," International Journal of Pure and Apllied Mathematics, vol. 112, no. 1, pp. 177-188, 2017.

[5] A. Kaewkhao and K. Neammanee, "Fixed point theorems of multivalued Zamfirescu mapping," Journal of Mathematics Research, vol. 2, pp. 150-156, 2010.

[6] E. Ameer, H. Aydi, M. Arshad, H. Alsamir, and M. S. Noorani, "Hybrid multivalued type contraction mappings in $\alpha \mathrm{K}$-complete partial b-metric spaces and applications," Symmetry, vol. 11, no. 1, p. 86, 2019.

[7] M. Joshi and A. Tomar, "On unique and nonunique fixed points in metric spaces and application to chemical sciences," Journal of Function Spaces, vol. 2021, Article ID 5525472, 11 pages, 2021.

[8] M. Joshiy, A. Tomar, and S. K. Padaliya, "Fixed point to fixed ellipse in metric spaces and discontinuous activation function," Applied Mathematics. E-Notes, vol. 21, pp. 225-237, 2021.

[9] V. Parvaneh, M. R. Haddadi, and H. Aydi, "On best proximity point results for some type of mappings," Journal of Function Spaces, vol. 2020, 6 pages, 2020. 
[10] H. Aydi, H. Lakzian, Z. D. Mitrovic, and S. Radenovic, "Best proximity points of MT-Cyclic contractions with property UC," Numerical Functional Analysis and Optimization, vol. 41, no. 7, pp. 871-882, 2020.

[11] E. Karapinar, S. Czerwik, and H. Aydi, “( $\alpha, \psi)$-Meir-Keeler contraction mappings in generalized b-metric spaces," Journal of Function Spaces, vol. 2018, Article ID 3264620, 4 pages, 2018.

[12] H. Kaneko, "Single and multivalued contractions," Bollettino dell'Unione Matematica Italiana, vol. 6, pp. 29-33, 1985.

[13] R. Kannan, "Some results on fixed points," Bulletin of the Calcutta Mathematical Society, vol. 60, pp. 71-76, 1968.

[14] S. Reich, "Some remarks concerning contraction mappings," Canadian Mathematical Bulletin, vol. 14, no. 1, pp. 121-124, 1971.

[15] H. Aydi, E. Karapinar, and A. F. R. L. de Hierro, "w-interpolative Ciric-Reich-Rus type contractions," Mathematics, vol. 7, no. 1, p. 57, 2019.

[16] S. Reich, "Remarks on fixed points," Rendiconti Lincei. Scienze Fisiche e Naturali, vol. 52, pp. 689-697, 1972.

[17] L. A. Zadeh, "Fuzzy sets," Information and Control, vol. 8, no. 3, pp. 338-353, 1965.

[18] A. Bajpai and V. S. Kushwah, "Importance of fuzzy logic and application areas in engineering research," International Journal of Recent Technology and Engineering, vol. 7, pp. 14671471, 2019.

[19] P. O. Mohammed, H. Aydi, A. Kashuri, Y. S. Hamed, and K. M. Abualnaja, "Midpoint inequalities in fractional calculus defined using positive weighted symmetry function kernels," Symmetry, vol. 13, no. 4, p. 550, 2021.

[20] K. Javed, F. Uddin, H. Aydi, A. Mukheimer, and M. Arshad, "Ordered-theoretic fixed point results in fuzzy b-metric spaces with an application," Journal of Mathematics, vol. 2021, Article ID 6663707, 7 pages, 2021.

[21] S. Fahad and A. Shah, "Intelligent testing using fuzzy logic," in Innovations in E-learning, Instruction Technology, Assessment, and Engineering Education, M. Iskander, Ed., pp. 95-98, Springer, Dordrecht, The Netherlands, 2007.

[22] N. Rusmiari, D. Putra, and A. Sasmita, "Fuzzy logic method for evaluation of diffculty level of exam and student graduation," International Journal of Computer Science, vol. 10, pp. 223229, 2013.

[23] A. Sobrino, "Fuzzy logic and education: teaching the basics of fuzzy logic through an example (by way of cycling)," Education in Science, vol. 3, no. 2, pp. 75-97, 2013.

[24] N. Mlaiki, N. Souayah, T. Abdeljawad, and H. Aydi, “A new extension to the controlled metric type spaces endowed with a graph," Advances in Differential Equations, vol. 2021, no. 1, p. 94, 2021.

[25] H. A. Hammad, H. Aydi, and Y. U. Gaba, "Exciting fixed point results on a novel space with supportive applications," Journal of Function Spaces, vol. 2021, Article ID 6613774, 12 pages, 2021.

[26] O. Kramosil and J. Michalek, "Fuzzy metric and statistical metric spaces," Kybernetika, vol. 11, pp. 336-344, 1975.

[27] M. Grabeic, "Fixed points in fuzzy metric spaces," Fuzzy Sets and Systems, vol. 27, no. 3, pp. 385-389, 1988.

[28] A. George and P. Veeramani, "On some results in fuzzy metric spaces,” Fuzzy Sets and Systems, vol. 64, no. 3, pp. 395-399, 1994.
[29] K. Javed, H. Aydi, F. Uddin, and M. Arshad, "On orthogonal partial b-metric spaces with an application," Journal of Mathematics, vol. 2021, Article ID 6692063, p. 7, 2021.

[30] R. Rana, R. C. Dimri, and A. Tomar, "Fixed point theorems in fuzzy metric spaces using implicit relations," International Journal of Computer Applications, vol. 8, no. 1, pp. 16-21, 2010.

[31] S. Beloul, A. Tomarb, and R. Sharma, "Weak subsequential continuity in fuzzy metric spaces and application," International Journal of Nonlinear Analysis and Applications, vol. 12, no. 2, pp. 1485-1486, 2021.

[32] Y. J. Cho, S. Sedghi, and N. Shobe, "Generalized fixed point theorems for compatible mappings with some types in fuzzy metric spaces," Chaos, Solitons \& Fractals, vol. 39, no. 5, pp. 2233-2244, 2009.

[33] R. K. Saini and V. Gupta, "Common coincidence points of Rweakly commuting fuzzy maps," Thai Journal of Mathematics, vol. 6, pp. 109-115, 2008.

[34] S. N. Mishra, S. N. Sharma, and S. L. Singh, "Common fixed points of maps on fuzzy metric spaces," International Journal of Mathematics and Mathematical Sciences, vol. 17, no. 2, pp. 253-258, 1994.

[35] V. Gregori and A. Sapena, "On fixed-point theorems in fuzzy metric spaces," Fuzzy Sets and Systems, vol. 125, no. 2, pp. 245-252, 2002.

[36] S. Manro and A. Tomar, "Faintly compatible maps and existence of common fixed points in fuzzy metric space," Annals of Fuzzy Mathematics and Informatics, vol. 8, no. 2, pp. 223230, 2014.

[37] S. L. Singh and A. Tomar, "Fixed point theorems in FM -spaces," The Journal of Fuzzy Mathematics, vol. 12, no. 4, pp. 845-859, 2004.

[38] R. Vasuki, "Common fixed point for R-weakly commuting maps in fuzzy metric space," Indian Journal of Pure and Applied Mathematics, vol. 30, pp. 419-423, 1999.

[39] I. Beg and M. Abbas, "Invariant approximation for fuzzy nonexpansive mappings," Mathematica Bohemica, vol. 136, no. 1, pp. 51-59, 2011.

[40] V. Popa, "Some fixed point theorems for compatible mappings satisfying an implicit relation," Demonstratio Mathematica, vol. 32, pp. 157-163, 1999.

[41] M. Imdad and J. Ali, "A general fixed point theorem in fuzzy metric spaces via an implicit function," Journal of Applied Mathematics \& Informatics, vol. 26, pp. 591-603, 2008.

[42] M. Imdad, K. Santosh, and M. S. Khan, "Remarks on some fixed point theorems satisfying implicit relations," Radovi Matematickz, vol. 11, pp. 135-143, 2002.

[43] X. Li, S. U. Rehman, S. U. Khan, H. Aydi, J. Ahmad, and N. Hussain, "Strong coupled fixed point results and applications to Urysohn integral equations," Dynamic Systems and Applications, vol. 30, pp. 197-218, 2021.

[44] S. U. Rehman, R. Chinram, and C. Boonpok, "Rational type fuzzy-contraction results in fuzzy metric spaces with an application," Journal of Mathematics, vol. 2021, 13 pages, 2021.

[45] B. Schweizer and A. Sklar, "Statical metric spaces," Pacific Journal of Mathematics, vol. 10, pp. 314-334, 1960.

[46] C. D. Bari and C. Vetro, "Fixed points, attractors and weak fuzzy contractive mappings in a fuzzy metric space," Journal of Fuzzy Mathematics, vol. 1, pp. 973-982, 2005. 